\title{
Colonização e infecção em pacientes com cateter peridural não tunelizado para analgesia*
}

\author{
Colonization and infection in patients with non-tunneled epidural catheter for analgesia \\ Colonización e infección en pacientes con catéter peridural no tunelizado para analgesia
}
Solange Diccini ${ }^{1}$, Maria Cecília Torres de Araújo Pimenta Ilsilara ${ }^{2}$, Mara Harumi Miyake $^{3}$, Angélica Gonçalves Silva Belasco', Dulce Aparecida Barbosa ${ }^{1}$

\section{RESUMO}

Objetivo: Correlacionar a colonização e a infecção no paciente em uso do cateter peridural não tunelizado para analgesia. Métodos: Estudo prospectivo realizado com 109 pacientes clínicos ou cirúrgicos e com tempo de permanência do cateter $\geq 72$ horas em um hospital privado do Município de São Paulo. A cultura de vigilância foi realizada com base na coleta da ponta do cateter peridural, swab pericateter e hemocultura periférica. Resultados: Dentre os 109 acompanhados 109 pacientes, 106 (97,2\%) foram cirúrgicos e 3 (2,8\%) clínicos. O tempo de permanência do cateter variou de 3 a 18 dias e a taxa de colonização foi de $11 \%$. Doze pacientes apresentaram colonização do cateter peridural e o principal micro-organismo isolado foi o Staphylococcus coagulase-negativa. Conclusões: A cultura positiva do cateter peridural constituiu um fator preditor para a colonização, porém nenhum paciente com positividade evoluiu com infecção.

Descritores: Analgesia epidural; Cuidados de enfermagem; Dor; Infecção/prevenção \& controle

\begin{abstract}
Objective: To correlate the colonization and infection in patients using non-tunneled epidural catheter for analgesia effects. Methods: This is a prospective study of 109 clinical or surgical patients, with catheter dwell time $>72$ hours, in a private hospital in the city of Sao Paulo. The culture of surveillance was carried out based on the collection of the epidural catheter tip, swab peri-catheter and peripheral blood cultures. Results: Among the 109 patients accompanied, $106(97.2 \%)$ were surgical and $3(2.8 \%)$ clinical. The catheter dwell time ranged from 3 to 18 days and the rate of colonization was $11 \%$. Twelve patients had epidural catheter colonization and the main organism isolated was coagulase-negative Staphylococcus. Conclusions: The positive culture of epidural catheter was a predictive factor for colonization; however, it was not found positive patients who evolved to infection.
\end{abstract}

Keywords: Epidural analgesia; Nursing care; Pain; Infection/prevention \& control

\section{RESUMEN}

Objetivo: Correlacionar la colonización y la infección, en pacientes que usan catéter peridural no tunelizado para efectos de analgesia. Métodos: Se trata de un estudio prospectivo realizado con 109 pacientes clínicos o quirúrgicos, con tiempo de permanencia del catéter $\geq 72$ horas, en un hospital privado del Municipio de Sao Paulo. La cultura de vigilancia fue realizada con base en la recolección de la punta del catéter peridural, swab pericatéter y hemocultura periférica. Resultados: Entre los 109 pacientes acompañados, 106 (97,2\%) fueron quirúrgicos y $3(2,8 \%)$ clínicos. El tiempo de permanencia del catéter varió de 3 a 18 días y la tasa de colonización fue de $11 \%$. Doce pacientes presentaron colonización del catéter peridural y el principal microorganismo aislado fue el Staphylococcus coagulase-negativa. Conclusiones: La cultura positiva del catéter peridural constituyó un factor de predicción para la colonización, sin embargo no se encontró ningún paciente con positividad que evolucionó para infección.

Descriptores: Analgesia epidural; Atención de enfermería; Dolor; Infección/ prevención \& control

\footnotetext{
* Trabalho extraído da dissertação de mestrado "Incidência de infecção em pacientes com cateter peridural para analgesia", apresentada a Escola Paulista de Enfermagem da Universidade Federal de São Paulo - UNIFESP - São Paulo (SP), Brasil.

${ }^{1}$ Professora Adjunto da Escola Paulista de Enfermagem da Universidade Federal de São Paulo- UNIFESP - São Paulo (SP), Brasil.

${ }^{2}$ Mestre em Ciências da Saúde pela Universidade Federal de São Paulo - UNIFESP - São Paulo (SP), Brasil.

${ }^{3}$ Pós-graduanda (Mestrado) em Enfermagem; Bolsista CAPES pela Escola Paulista de Enfermagem da Universidade Federal de São Paulo - UNIFESP São Paulo (SP), Brasil.
} 


\section{INTRODUÇÃO}

A dor pós-operatória desencadeia uma resposta de estresse que ativa o sistema nervoso autônomo e provoca vários efeitos adversos no organismo. Uma analgesia adequada auxilia na recuperação do paciente, proporcionando melhor evolução clínica e alta hospitalar precoce $^{(1-5)}$.

Em cirurgias de grande porte e de longa duração, a anestesia peridural é geralmente, associada à anestesia geral e o cateter peridural pode ser mantido no pós-operatório para infusão de solução analgésica ao paciente ${ }^{(4-5)}$. O cateter peridural utilizado no pós-operatório é de curta permanência, ficando em média, quatro dias ou enquanto houver dor que justifique a utilização de analgésicos por essa via.

Embora seja um método muito difundido e tenha revolucionado a qualidade de analgesia oferecida aos pacientes ${ }^{(4-6)}$, há vários relatos de infecções diretamente relacionadas à via peridural para administração de analgésicos ${ }^{(7-8)}$.

A incidência de infecção relacionada ao cateter peridural varia entre $0,06 \%$ a $5,3 \%$ em estudos com pacientes cirúrgicos ${ }^{(9-10)}$.

No espaço peridural, a infecção, ou seja, profunda, varia entre $0 \%$ e $0,7 \% \%^{(7-9)}$. No entanto, é a complicação mais preocupante, pois pode evoluir para a formação de abscesso peridural ${ }^{(3,11-15)}$, mas a incidência de abscesso peridural espontâneo pode ser muito baixa, entre $0,002 \%$ e $0,012 \% \%^{(12-14,16)}$. Quando relacionada à anestesia ou analgesia peridural está em torno de 5,5\% $\%^{(13)}$. Trata-se de uma situação grave que frequentemente provoca lesões neurológicas irreversíveis ${ }^{(3,12,14)}$. Além da infecção, outras complicações relacionadas ao cateter peridural são: a desconexão da extremidade externa, a migração do cateter para o espaço subaracnoideo, a saída acidental, a dobra e a quebra do cateter ${ }^{(5,10)}$.

Estudos sobre a utilização do cateter peridural para analgesia têm demonstrado que as taxas de colonização ou contaminação ainda se mantêm altas, variando de $0 \%$ a $28,8 \% \%^{(3,9)}$. A colonização do cateter pela pele e a disseminação hematogênica de um processo infeccioso distante são as principais vias de contaminação para o cateter, peridural ${ }^{(11-13)}$. Outros fatores são a contaminação durante a implantação do cateter, a administração de soluções analgésicas contaminadas, a migração de agentes patógenos por capilaridade pelo cateter exteriorizado ou a formação do biofilme sobre o material implantado ${ }^{(3,9)}$.

A idade e a gravidade da doença de base são os fatores predisponentes individuais, e o tempo de permanência do cateter é o fator determinante para o risco de infecção relacionada ao cateter peridural, aumentando proporcionalmente, de acordo com a permanência do cateter inserido ${ }^{(3,9)}$.
Os micro-organismos isolados com mais frequência na ponta e na inserção do cateter peridural são o Staphylococcus coagulase-negativa, destacando-se o Staphylococcus epidermidis; o Staphylococcus aureus e o Pseudomonas aeruginosa ${ }^{(3,9-19)}$.

Atualmente, o tratamento criterioso da dor é uma realidade na maioria dos hospitais, sendo imprescindível que os profissionais de saúde conheçam os vários métodos disponíveis para analgesia. A via peridural é a preferencialmente escolhida para o alívio da dor intensa, mas ainda há muitas dúvidas em relação aos reais riscos de colonização e infecção no uso do cateter peridural para analgesia. Vários estudos demonstraram a eficácia e os benefícios desta via para infusão de solução analgésica, porém as diretrizes utilizadas para os cuidados de enfermagem são baseadas nos cateteres vasculares. Frente ao exposto e à grande frequência com que o cateter peridural vem sendo utilizado para o tratamento da dor aguda, este estudo teve como objetivo correlacionar a colonização e a infecção no paciente em uso do cateter peridural não tunelizado para analgesia.

\section{MÉTODOS}

Estudo prospectivo realizado nas unidades de internação e de terapia intensiva de um hospital da rede privada do Município de São Paulo, de porte médio, no período de setembro de 2003 a março de 2005. O projeto de pesquisa foi aprovado pelo Comitê de Ética e Pesquisa do hospital, como processo do CEP n ${ }^{\circ}$ 0364/ 04 e todos os pacientes incluídos assinaram o Termo de Consentimento Livre e Esclarecido.

Para a coleta de dados, foi desenvolvido um instrumento, com as seguintes informações dados: sóciodemográficos (sexo, idade, cor, escolaridade); de internação (diagnóstico clínico ou cirúrgico, comorbidades, antibioticoterapia); referentes ao cateter peridural: nível de inserção; técnica utilizada: analgesia controlada pelo paciente por bomba de infusão ou analgesia intermitente com injeção de bólus de solução analgésica, utilizando-se seringa; queixas do paciente relacionadas ao cateter peridural; presença de sinais de infecção no local da inserção ou sistêmica; remoção do cateter (tempo de permanência em dias e motivo da retirada do cateter peridural); coleta da ponta do cateter e swab de secreção na vigência de sinais flogísticos pericateter e resultado de culturas.

O protocolo do estudo foi realizado com as seguintes etapas: a indicação da analgesia peridural seguiu os critérios clínicos da equipe do centro de dor do hospital e/ou da equipe responsável pelo paciente; a implantação do cateter peridural foi realizada com técnica asséptica (escovação das mãos com antisséptico degermante, barreira de precaução máxima e a antissepsia da pele do 
paciente com solução de povinilpirrolina-iodo aquosa a $1 \%$ pelo anestesista no centro cirúrgico ou à beira do leito em pacientes da unidade de terapia intensiva, com impossibilidade de transporte. O cateter peridural ou caudal foi escolhido para analgesia durante o pósoperatório; o curativo no local da punção foi feito com fita adesiva micropore ${ }^{\circledR}$ ou com curativo transparente bioclusive $^{\circledR}$ (toda a extensão do cateter que ficava exteriorizada, foi fixada com fita adesiva micropore ${ }^{\circledR}$ na região dorsal e o curativo não foi trocado durante o tempo de permanência do cateter, em razão ao risco de deslocamento); a utilização de antimicrobianos ocorreu nos pacientes cirúrgicos e nos pacientes clínicos somente na vigência de infecção diagnosticada.

Durante o período do estudo, foram acompanhados 254 pacientes recebendo analgesia pelo cateter peridural e destes 145 (57\%) foram excluídos: $89(61,4 \%)$ pacientes em razão do tempo de permanência do cateter inferior a 72 horas, $16(11 \%)$ pacientes por saída acidental do cateter, $35(24,1 \%)$ pacientes pela técnica de tunelização (cateter peridural tunelizado) e $5(3,5 \%)$ pacientes por perda do seguimento por retirada do cateter pela equipe responsável pelo paciente. Os $109(43 \%)$ pacientes que tiveram cateter peridural implantado sem tunelização (cateter peridural não tunelizado) foram incluídos neste estudo.

Diariamente, os pacientes foram acompanhados durante o uso do cateter, por um anestesista e pela enfermeira do centro de dor, sendo avaliados em relação: ao curativo; ao aspecto da pele no local da inserção, às conexões do cateter e as queixas de dor. A retirada do cateter peridural e a coleta de material foram feitas pelo anestesista ou pelo médico responsável pelo paciente. A cultura da ponta do cateter peridural foi realizada em todos os pacientes e na vigência de sinais flogísticos com presença de secreção, foi realizada também a coleta de swab e a hemocultura. Esta era colhida, em caso de temperatura acima de $38^{\circ} \mathrm{C}$. O laboratório do hospital foi responsável pelo envio dos resultados das culturas de cada paciente e o método laboratorial respeitou as diretrizes do The National Committee for Clinical Laboratory Standards ${ }^{(20)}$ desde a coleta até o resultado dos espécimes analisados.

A análise estatística foi feita pela inserção de dados no programa do software Microsoft Excel, onde as variáveis numéricas foram apresentadas com valores da média, desvio-padrão, mediana e variação com valores mínimos e máximos de cada uma delas. As variáveis categóricas foram analisadas pelos testes de Qui-quadrado ou exato de Fisher. Os resultados foram considerados estatisticamente significantes para o valor de $p \leq 0,05$.

\section{RESULTADOS}

Em relação à caracterização da amostra, houve um predomínio de pacientes do sexo masculino (55\%), com a mediana de idade de 57 anos variando de 25 a 81 anos. Nas pacientes do sexo feminino, a mediana da idade foi de 59 anos, variando de 21 a 87 anos. Em relação à cor, ocorreu predomínio da branca $(94,5 \%)$ e quanto ao nível de instrução, a maioria dos pacientes tinha o nível superior de escolaridade (45\%). As comorbidades mais frequentes nos $106(97,2 \%)$ pacientes cirúrgicos foram a Hipertensão arterial sistêmica $(11,4 \%)$, a Diabetes mellitus $(8,5 \%)$ e a associação entre diabéticos e hipertensos (8,5\%). Dos $3(2,8 \%)$ pacientes internados para tratamento clínico com cateter peridural não tunelizado, dois eram diabéticos (Tabela 1).

Tabela 1 - Caracterização dos pacientes submetidos à analgesia peridural com cateteres não tunelizados - São paulo, 09/2003 a 03/2005

\begin{tabular}{|c|c|c|}
\hline Caracterização & n..$^{\circ}$ & $\%$ \\
\hline \multicolumn{3}{|l|}{ Sexo } \\
\hline Masculino & 60 & 55,0 \\
\hline Feminino & 49 & 45,0 \\
\hline \multicolumn{3}{|l|}{ Cor } \\
\hline Branca & 103 & 94,5 \\
\hline Não branca & 6 & 5,5 \\
\hline \multicolumn{3}{|l|}{ Nível de instrução } \\
\hline Ensino Fundamental & 24 & 22,0 \\
\hline Ensino Médio & 36 & 33,0 \\
\hline Superior & 49 & 45,0 \\
\hline \multicolumn{3}{|l|}{ Comorbidades } \\
\hline Diabetes mellitus & 11 & 10,1 \\
\hline Hipertensão arterial sistêmica & 12 & 11,4 \\
\hline Diabetes mellitus e hipertensão arterial & 9 & 8,5 \\
\hline
\end{tabular}

DP - desvio padrão

Dos 109 pacientes, $106(97,2 \%)$ utilizaram o cateter peridural para tratamento cirúrgico, sendo mantido no pós-operatório para analgesia e três pacientes $(2,8 \%)$ foram internados para tratamento clínico da dor aguda. Todos os pacientes cirúrgicos utilizaram antibioticoprofilaxia no pós-operatório determinada pela equipe cirúrgica e os pacientes clínicos receberam antibioticoterapia somente na vigência de infecção.

Dos três pacientes que foram internados para tratamento clínico, dois tiveram inserção lombar e um inserção torácica, com uma mediana de sete dias, variando de 7 a 11 dias de permanência. Dos 106 pacientes cirúrgicos, $56(52,8 \%)$ tiveram inserção lombar, com uma mediana de cinco dias, variando de 3 a 18 dias de permanência e $50(47,2 \%)$ pacientes com inserção torácica, com mediana de cinco dias, variando de 3 a 18 dias de permanência (Tabela 2). Não houve diferença estatisticamente significante entre o tempo de permanência e o local de inserção do cateter peridural não tunelizado.

Todos os 109 pacientes acompanhados que utilizaram o cateter peridural não tunelizado receberam analgesia controlada pelo paciente por bomba de infusão. Destes pacientes, $6(5,5 \%)$ queixaram-se de parestesia no membro 
inferior, sendo reprogramada a bomba de infusão, não ocorrendo outras intercorrências. O alívio da dor foi considerado satisfatório por todos os pacientes e o cateter peridural não tunelizado foi retirado ao término do tratamento da dor aguda.

Tabela 2 - Pacientes segundo tempo de permanência do cateter peridural não tunelizado em relação ao nível de inserção - São Paulo 09/2003 a 03/2005

\begin{tabular}{|c|c|c|c|c|c|c|c|}
\hline \multirow{3}{*}{$\begin{array}{l}\text { Tempo de } \\
\text { Permanência } \\
\text { (dias) }\end{array}$} & \multicolumn{4}{|c|}{ Inserção } & \multicolumn{2}{|c|}{ Total } & \multirow{3}{*}{$\begin{array}{c}\text { Valor } \\
\text { de } \\
p^{*}\end{array}$} \\
\hline & \multicolumn{2}{|c|}{ Torácico } & \multicolumn{2}{|c|}{ Lombar } & \multirow{2}{*}{ n..$^{o}$} & \multirow{2}{*}{$\%$} & \\
\hline & $\mathbf{n}$ & $\%$ & $\mathbf{n}$ & $\%$ & & & \\
\hline 3 & 8 & 7,3 & 12 & 11,0 & 20 & 18,3 & 0,32 \\
\hline 4 & 6 & 5,5 & 11 & 10,1 & 17 & 15,6 & 0,19 \\
\hline 5 & 13 & 11,9 & 13 & 11,9 & 26 & 23,8 & 0,82 \\
\hline 6 & 10 & 9,2 & 4 & 3,7 & 14 & 12,8 & 0,16 \\
\hline 7 & 10 & 9,2 & 4 & 3,7 & 14 & 12,8 & 0,16 \\
\hline 8 & 1 & 0,9 & 2 & 1,8 & 3 & 2,7 & 0,60 \\
\hline 9 & 5 & 4,6 & 2 & 1,8 & 7 & 6,4 & 0,44 \\
\hline 10 & 3 & 2,6 & 1 & 0,9 & 4 & 3,7 & 0,62 \\
\hline 11 & 1 & 0,9 & 2 & 1,8 & 3 & 2,7 & 0,60 \\
\hline 18 & - & - & 1 & 0,9 & 1 & 0,9 & 0,48 \\
\hline Total & 57 & 52,3 & 52 & 47,7 & 109 & 100 & \\
\hline
\end{tabular}

* Teste exato de Fisher

A hiperemia no local da inserção do cateter peridural não tunelizado ocorreu em 3 (2,7\%) dos 109 pacientes acompanhados.

Dentre estes, $12(11 \%)$ tiveram os cateteres colonizados, e sete cateteres $(58,4 \%)$ tiveram a inserção ao nível torácico e $5(41,6 \%)$ ao nível lombar. Dos pacientes que apresentaram colonização no cateter, não houve casos de infecção.

Tabela 3 - Característica da colonização da ponta do cateter peridural em relação ao nível de inserção em pacientes, São Paulo 09/2003 a 03/2005

\begin{tabular}{|c|c|c|c|c|c|c|}
\hline \multirow{3}{*}{$\begin{array}{l}\text { Micro-organismos } \\
\text { isolados }\end{array}$} & \multicolumn{4}{|c|}{ Nível de inserção } & \multicolumn{2}{|c|}{ Total } \\
\hline & \multicolumn{2}{|c|}{ Torácico } & \multicolumn{2}{|c|}{ Lombar } & \multirow[b]{2}{*}{$\mathbf{n}$} & \multirow{2}{*}{$\%$} \\
\hline & $\mathbf{n}$ & $\%$ & $\mathbf{n}$ & $\%$ & & \\
\hline & 5 & 41,8 & 4 & 33,3 & 9 & 75,1 \\
\hline Staphylococcus aureus & & 8,3 & 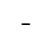 & - & & 8,3 \\
\hline & 1 & 8,3 & - & - & & 8,3 \\
\hline Acinetob & & - & 1 & 8,3 & 1 & 8,3 \\
\hline Total & & 58,4 & & 41,6 & 12 & 100 \\
\hline
\end{tabular}

O tempo médio de permanência do cateter peridural de inserção torácica foi de 5,9 dias e de inserção lombar foi de 5,4 dias Quanto ao tempo de ocorrência da colonização, observou-se que os cateteres de inserção torácica colonizaram entre o $3^{\circ}$ até o $10^{\circ}$ dia de permanência, e os cateteres de inserção lombar colonizaram entre o $5^{\circ}$ até o $18^{\circ}$ dia de permanência.

Dos pacientes que apresentaram colonização nos cateteres, o Staphylococcus coagulase-negativa foi o micro-organismo que predominou, tanto no nível de inserção torácico como lombar. O Staphylococcus aureus, o Enterococcus cloacae e o Acinetobacter baumanii foram os micro-organismos encontrados na colonização dos cateteres peridurais (Tabela 3 ).

Um dos pacientes que apresentou colonização por Staphylococcus coagulase negativa, faleceu por sepse pulmonar (broncopneumonia) que não foi relacionada pela equipe médica ao cateter peridural não tunelizado.

Quando foram relacionados os pacientes que apresentaram colonização e não colonização dos cateteres peridurais não tunelizados com os fatores de risco: sexo, idade, comorbidades e nível de inserção do cateter, não houve relação estatisticamente significante com estes fatores (Tabela 4).

Tabela 4 - Pacientes segundo principais fatores de risco relacionados à colonização do cateter peridural não tunelizado - São Paulo, 09/2003 a 03/2005

\begin{tabular}{|c|c|c|c|c|c|c|c|}
\hline \multirow[b]{2}{*}{$\begin{array}{l}\text { Fatores de } \\
\text { risco }\end{array}$} & \multicolumn{3}{|c|}{ Colonização } & \multicolumn{3}{|c|}{ Não colonização } & \multirow{2}{*}{$\begin{array}{c}\text { Valor } \\
\text { de } \\
P^{*}\end{array}$} \\
\hline & $\mathbf{n}$ & $\%$ & Total & $\mathbf{n}$ & $\%$ & Total & \\
\hline \multicolumn{8}{|l|}{ Sexo } \\
\hline Masculino & 6 & 50,0 & 12 & 54 & 55,7 & 97 & 0,94 \\
\hline Feminino & 6 & 50,0 & & 43 & 44,3 & & \\
\hline \multicolumn{8}{|l|}{ Idade } \\
\hline$<40$ anos & 2 & 16,6 & 12 & 13 & 13,4 & 97 & 0,67 \\
\hline$>40$ anos & 10 & 83,4 & & 84 & 86,6 & & \\
\hline \multicolumn{8}{|l|}{ Comorbidade } \\
\hline Presente & 6 & 50,0 & 12 & 22 & 22,7 & 97 & 0,07 \\
\hline Ausente & 6 & 50,0 & & 75 & 77,3 & & \\
\hline \multicolumn{8}{|c|}{ Inserção cateter } \\
\hline Torácica & 7 & 58,3 & 12 & 50 & 51,5 & 97 & 0,89 \\
\hline Lombar & 5 & 41,7 & & 47 & 48,5 & & \\
\hline
\end{tabular}

* teste exato de Fisher

\section{DISCUSSÃO}

A população estudada constituiu-se de pacientes cirúrgicos e clínicos de ambos os sexos, com idade média de 57, 5 anos. O cateter peridural não tunelizado foi utilizado para tratamento da dor aguda, sendo inserido aos níveis torácico ou lombar, com tempo de permanência, variando entre 3 e 18 dias, e a técnica utilizada foi a analgesia controlada pelo paciente (ACP) por bomba de infusão.

É importante considerar que, em decorrência da manipulação do cateter peridural não tunelizado para analgesia, os pacientes podem apresentar colonização ou complicações infecciosas e não infecciosas relacionadas a seu uso.

Em nosso estudo, a parestesia do membro inferior $(5,5 \%)$ foi a única intercorrência não infecciosa relacionada à infusão de solução analgésica por ACP, e a parestesia transitória é menor do que 10\% quando associada à analgesia peridural ${ }^{(21)}$.

Não ocorreram casos de infecção, porém a taxa de colonização relacionada ao cateter não tunelizado para este estudo, foi de $11 \%$. Dados da literatura mostram 
que a taxa de colonização varia entre $0 \%$ e $28,8 \%{ }^{(3,9,16)}$. Quanto aos sinais flogísticos, a hiperemia no local da inserção do cateter peridural não tunelizado ocorreu em $3(2,7 \%)$ dos 109 pacientes, sendo o único sinal apresentado pelos pacientes, além da positividade da cultura na ponta do cateter peridural.

Em relação à colonização na ponta do cateter peridural, o principal micro-organismo isolado foi o Staphylococcus coagulase-negativa, semelhante aos outros estudos já desenvolvidos na literatura ${ }^{(3,9-18,22)}$. É importante considerar que, o Staphylococcus coagulase-negativa é o agente etiológico mais frequente na infecção relacionada ao cateter de curta permanência. Tem baixa patogenicidade e pode ser considerado também um colonizante, representando a própria flora residente da pele ${ }^{(3,6,11-16)}$.

Para este estudo, o Staphylococcus coagulase-negativa, o Staphylococcus aureus, o Acinetobacter baumanii e o Enterobacter cloacae foram considerados colonizantes de pele, já que não houve sinais locais ou sistêmicos de infecção. Um estudo prospectivo realizado com 1.443 pacientes, demonstrou que $28,8 \%$ dos pacientes obtiveram cultura positiva do cateter peridural por, pelo menos, um microorganismo, sugerindo que há uma significante proporção de cultura positiva representando a colonização da ponta do cateter peridural pela pele e sua contaminação durante a retirada, sem relevância clínica para infecção ${ }^{(23)}$.

Os principais fatores de risco que contribuem para a colonização do cateter peridural são: idade $\geq 40$ anos, presença de comorbidades, internação em unidade de cuidados intensivos, sexo masculino, tempo de permanência $\geq 48$ horas do cateter peridural, uso de soluções hipertônicas e ausência de administração da antibioticoprofilaxia cirúrgica ${ }^{(16,24-25)}$.

A colonização do cateter peridural ocorreu em ambos os sexos, em pacientes com idade $\geq 40$ anos $(83,4 \%)$ e $50 \%$ dos pacientes colonizados tinham comorbidade associada, como Hipertensão arterial e Diabetes mellitus. De acordo com a literatura, a Diabetes mellutus é um fator que aumenta a predisposição para a colonização do cateter peridural não tunelizado ${ }^{(3,9,25)}$.

A inserção do cateter não tunelizado ao nível torácico foi considerada, como fator de maior risco de infecção no espaço peridural, quando comparada com o nível lombar ${ }^{(6,18,22,26)}$. Em nosso estudo, não ocorreram infecções, mesmo quando a colonização relacionada ao cateter não tunelizado foi maior na região torácica em relação à lombar. A literatura mostra que a colonização do cateter peridural é mais frequente quando inserida em região lombar ${ }^{(11,21)}$, o que não foi encontrado neste estudo.

Quanto ao tempo de permanência e de colonização, os cateteres de inserção torácica colonizaram entre o $3^{\circ}$ até o $10^{\circ}$ dia de permanência, e os cateteres de inserção lombar colonizaram entre o $5^{\circ}$ até o $18^{\circ}$ dia de permanência. Em um estudo prospectivo realizado, a colonização do cateter ocorreu a partir do $3^{\circ}$ dia de inserção, atingindo uma taxa de $10 \%$ de colonização até o $7^{\circ}$ dia de permanência do cateter peridural ${ }^{(16)}$.

O tempo médio de permanência do cateter peridural de inserção torácica foi de 5,9 dias e de inserção lombar foi de 5,4 dias. O tempo de permanência acima de 48 horas em associação ao uso de soluções hipertônicas pode contribuir com a colonização do cateter peridural ${ }^{(25)}$. É fundamental que seja avaliada a necessidade de permanência do cateter para que seja removido tão logo termine sua indicação, pois quanto maior o tempo de permanência, maior é o risco de colonização e infecção $\mathrm{O}^{(3,9,16,25)}$. Em nosso estudo, a retirada do cateter peridural foi realizada de acordo com o término da indicação de analgesia por esta via, como terapêutica da dor aguda.

Em nosso estudo, a idade, a comorbilidade, o sexo, o nível de inserção e o tempo de permanência do cateter não foram estatisticamente significantes, como fatores preditores da colonização do cateter peridural.

Considerando, os avanços tecnológicos para analgesia peridural na promoção do alívio da dor com poucos efeitos colaterais e melhoria da qualidade de vida do paciente, a ausência de infecção é uma situação desejada para esta técnica, como terapêutica da dor aguda. Nosso estudo demonstrou que a aplicação das boas práticas tanto da equipe médica como da equipe de enfermagem tem como resultado, uma taxa negativa de infecção relacionada ao cateter peridural, porém a colonização ainda é elevada e provalvemente, está relacionada com a manipulação desse cateter durante sua permanência no paciente como terapêutica da dor aguda.

Para reduzir o risco de colonização ou contaminação do cateter peridural, as seguintes medidas devem ser adotadas: escovação das mãos, uso da barreira de precaução máxima pelo anestesista; preparo da pele do paciente com antisséptico alcoólico na implantação do cateter; uso de curativo semiperméavel na inserção do cateter; uso de filtro antibacteriano durante a permanência do cateter peridural no paciente; vigilância do curativo e do local de inserção do cateter na pele, controle da temperatura do paciente e monitorização das queixas do paciente, como a ocorrência de sinais de meningismo ${ }^{(18-19,22-24,27-30)}$.

Neste contexto, a atuação do enfermeiro é fundamental na educação e na supervisão da equipe de enfermagem em relação a todas as etapas que envolvem a manutenção do cateter peridural, ou seja, da implantação até a coleta do material para a cultura. A existência de protocolos de enfermagem e o treinamento da equipe garantem que estas medidas sejam eficazes para um controle mais rigoroso nos processos de prevenção da colonização ou de infecção relacionada ao uso do cateter peridural não tunelizado para analgesia. 


\section{CONCLUSÕES}

A analgesia peridural é um método seguro e eficaz, sendo excelente para o alívio da dor aguda.

A taxa de colonização relacionada ao uso do cateter

\section{REFERÊNCIAS}

1. Andrade MP. Dor pós-operatória: conceitos básicos de fisiopatologia e tratamento. Rev Dor. 2000;2(2):7-14.

2. Buggy DJ, Smith G. Epidural anaesthesia and analgesia: better outcome after major surgery?. Growing evidence suggests so. BMJ. 1999;319(7209):530-1.

3. Dawson S. Epidural catheter infections. J Hosp Infect. 2001;47(1):3-8.

4. Yeager MP, Glass DD, Neff R, Brinck-Johnsen T. Epidural anesthesia and analgesia in high-risk surgical patients. Anesthesiology. 1987;66(6):729-36.

5. Liu S, Carpenter RL, Neal JM. Epidural anesthesia and analgesia. Their role in postoperative outcome. Anesthesiology. 1995;82(6):1474-506. Comment in: Anesthesiology. 1995;83(5):1132.

6. Kindler CH, Seeberger MD, Staender SE. Epidural abscess complicating epidural anesthesia and analgesia. An analysis of the literature. Acta Anaesthesiol Scand. 1998;42(6):61420. Comment in: Acta Anaesthesiol Scand. 1998;42(6):60913.

7. Holt HM, Andersen SS, Andersen O, Gahrn-Hansen B, Siboni K. Infections following epidural catheterization. J Hosp Infect. 1995;30(4):253-60. Comment in: J Hosp Infect. 1997;35(3):245.

8. Brooks K, Pasero C, Hubbard L, Coghlan RH. The risk of infection associated with epidural analgesia. Infect Control Hosp Epidemiol. 1995;16(12):725-8.

9. Dawson SJ, Small H, Logan MN, Geringer S. Case control study of epidural catheter infections in a district general hospital. Commun Dis Public Health. 2000;3(4):300-2.

10. Burstal R, Wegener F, Hayes C, Lantry G. Epidural analgesia: prospective audit of 1062 patients. Anaesth Intensive Care. 1998;26(2):165-72. Comment in: Anaesth Intensive Care. 1998;26(5):597-8.

11. Kostopanagiotou G, Kyroudi S, Panidis D, Relia P, Danalatos A, Smyrniotis V, et al. Epidural catheter colonization is not associated with infection. Surg Infect (Larchmt). 2002;3(4):359-65.

12. Reihsaus E, Waldbaur H, Seeling W. Spinal epidural abscess: a meta-analysis of 915 patients. Neurosurg Rev. 2000;23(4):175-204; discussion 205.

13. Simons R, Dinner L, Lappin S. Skin abscess obscured by epidural catheter fixation. Anaesthesia. 2007;62(4):418.

14. Abreu MP, Deda RG, Cangiani LH, Aquino HMD, Ortiz J. Abscesso peridural após analgesia controlada pelo paciente por via peridural: relato de caso. Rev Bras Anestesiol. 2004;54(1):78-83.

15. Yuan HB, Zuo Z, Yu KW, Lin WM, Lee HC, Chan KH. Bacterial colonization of epidural catheters used for shortterm postoperative analgesia: microbiological examination and risk factor analysis. Anesthesiology. 2008;108(1):130-7.

16. Grewal S, Hocking G, Wildsmith JA. Epidural abscesses. Br J Anaesth. 2006;96(3):292-302. Comment in: Br J Anaesth. 2006;97(1):115-6.

17. O’Grady NP, Alexander M, Dellinger EP, Gerberding JL, peridural não tunelizado foi de $11 \%$, e o principal agente etiológico encontrado foi o Staphylococcus coagulase- negativa. Nos pacientes que apresentaram colonização da ponta do cateter, não ocorreu infecção relacionada ao uso do cateter peridural não tunelizado para analgesia.

Heard SO, Maki DG, et al. Guidelines for the prevention of intravascular catheter-related infections. Centers for Disease Control and Prevention. MMWR Recomm Rep. 2002;51(RR-10):1-29.

18. Steffen P, Seeling W, Essig A, Stiepan E, Rockemann MG. Bacterial contamination of epidural catheters: microbiological examination of 502 epidural catheters used for postoperative analgesia. J Clin Anesth. 2004;16(2): 927.

19. von Eiff C, Proctor RA, Peters G. Coagulase-negative staphylococci. Pathogens have major role in nosocomial infections. Postgrad Med. 2001;110(4):63-4, 69-70, 73-6.

20. The National Committee for Clinical Laboratory Standards. Methods for antimicrobial susceptibility testing/M11-A5. Wayne, PA: NCCLS; 2001.

21. Chan YC, Dasey N. Iatrogenic spinal epidural abscess. Acta Chir Belg. 2007;107(2):109-18.

22. Misiolek H, Wilkowska J, Werner M, Wojciechowski G, Kucia H, Grzadziel M, Styrkosz J. Bacteriological assessment of tip cultures of epidural catheters and the incidence of local infections during thoracic epidural analgesia - preliminary report. Pol J Cardiothorac Surg. 200;4(3):278-85.

23. Simpson RS, Macintyre PE, Shaw D, Norton A, McCann JR, Tham EJ. Epidural catheter tip cultures: results of a 4year audit and implications for clinical practice. Reg Anesth Pain Med. 2000;25(4):360-7.

24. Morin AM, Kerwat KM, Klotz M, Niestolik R, Ruf VE, Wulfi $\mathrm{H}$, et al. Risk factors for bacterial catheter colonization in regional anaesthesia. BMC Anesthesiol. 2005;5(1):1.

25. Capdevila X, Pirat P, Bringuier S, Gaertner E, Singelyn F, Bernard N, Choquet O, Bouaziz H, Bonnet F; French Study Group on Continuous Peripheral Nerve Blocks. Continuous peripheral nerve blocks in hospital wards after orthopedic surgery: a multicenter prospective analysis of the quality of postoperative analgesia and complications in 1,416 patients. Anesthesiology. 2005;103(5):921-3. Comment in: Anesthesiology. 2006;104(6):1348.

26. Mishra S, Bhatnagar S, Srikanti M, Gupta D. Clinical implication of routine bacterial culture from epidural catheter tips in postoperative cancer patients: a prospective study. Anaesthesia. 2006;61(9):878-82. Comment in: Anaesthesia. 2007;62(2):197-8.

27. Phillips JM, Stedeford JC, Hartsilver E, Roberts C. Epidural abscess complicating insertion of epidural catheters. $\mathrm{Br} \mathrm{J}$ Anaesth. 2002;89(5):778-82. Comment in: Br J Anaesth. 2003;90(5):706-7; author reply 707. Br J Anaesth. 2004;92(2):294-5.

28. Kasuda H, Fukuda H, Togashi H, Hotta K, Hirai Y, Hayashi M. Skin disinfection before epidural catheterization: comparative study of povidone-iodine versus chlorhexidine ethanol. Dermatology. 2002;204 Suppl 1: 42-6.

29. Rohrbach M, Plötz J. [Epidural abscess following delivery with peridural analgesia. The question of prevention]. Anaesthesist. 2001;50(6):411-5. German. 